Artículo

\title{
Reparto de utilidades en asociaciones temporales de inversionistas de tierra capital y maquinaria en la agricultura
}

\author{
Marcos Portillo Vázquez ${ }^{1}$ \\ Dora Ma. Sangerman-Jarquín ${ }^{2 \S}$ \\ Rocío Ramírez Jaspeado ${ }^{1}$ \\ ${ }^{1}$ Posgrado de Economía Agrícola-Universidad Autónoma Chapingo. Carretera México-Texcoco km 38.5, \\ Chapingo, Estado de México. CP. 56230. (mportillo49@yahoo.com.mx; ramirezrocio67@ hotmail.com). \\ ${ }^{2}$ Campo Experimental Valle de México-INIFAP. ${ }^{2}$ Carretera Los Reyes-Texcoco km 13.5, Coatlinchan, \\ Texcoco, Estado de México, México. CP. 56250. Tel. 01800 0882222, ext. 85353. \\ ${ }^{\S}$ Autora para correspondencia: sangerman.dora@inifap.gob.mx.
}

\section{Resumen}

En el medio rural existen los recursos necesarios y suficientes para obtener mucho mayores rendimientos que los que actualmente se obtienen. El gran problema de que no se aprovechen las potencialidades es que los principales recursos productivos se encuentran por separado en posesión de diferentes personas. El objetivo del manuscrito es sugerir un procedimiento matemático para identificar la aportación que cada uno de los recursos productivos que genera en el valor de la producción de los bienes agrícolas que se obtienen, el estudio se llevó a cabo en 2018. El procedimiento de reparto de utilidades se basa en un método de programación lineal que además de calcular con exactitud los montos adecuados del valor del producto entre los poseedores de recursos productivos, previamente realiza una recomendación para señalar en qué medida debe programarse cada uno de los cultivos posibles de llevarse a cabo, conociendo la magnitud de cada uno de los recursos se requiere para cultivar una hectárea en cada uno de los cultivos factibles y considerando la cantidad total de cada uno de los recursos que se encuentran disponibles. Los resultados encontrados, es que favorecen que la distribución de lo generado, resulte ser el justo y apropiado reparto de utilidades. Las conclusiones datos a procesar lo constituyen los precios de cada uno de los productos en el mercado y su valor monetario generado en una hectárea de cultivo; así como, este modelo constituye una buena salida para activar en las mejores condiciones a los programas del Gobierno Federal de mecanización del campo rural que no han dado los resultados esperados porque la maquinaria pertenece a un pequeño grupo de productores y éstos tienen serias dificultades para emplear toda su capacidad porque tienen poca tierra y el resto de productores no tienen recursos para tomarlos en renta.

Palabras clave: bienes agrícolas, precios, procedimiento matemático, recursos.

Recibido: abril de 2020

Aceptado: agosto de 2020 


\section{Introducción}

En muchas áreas agrícolas del país existen situaciones donde los productores agrícolas tienen tierra, pero no tienen maquinaria ni capital suficiente para explorarla con tecnologías de alto rendimiento (Romero, 1982). También existen productores que cuentan con maquinaria agrícola y muy poca tierra de tal forma que para aprovechar la maquinaria que se posee tratan de sacarle provecho rentando el servicio de labores con maquinaria para tratar de cubrir el alto costo invertido para aprovechar la capacidad ociosa, esta situación es verdaderamente preocupante cuando se ve que la existencia de tractores en el campo es mucho mayor de lo necesario como lo revelan censos de maquinaria agrícola, pero éstos están en manos de pocas personas que en relación a la tierra que poseen cada uno de estos productores, ésta les resulta mucho muy reducida para explotar la capacidad de la maquinaria que poseen (Guerrero, 2011).

En no muy pocos casos se presenta la situación de que la explotación agrícola aun entre los que poseen maquinaria, las siembras se realizan con insumos que no son los más productivos y modernos por falta de capital para invertir en las cantidades necesarias para elevar la productividad del recurso tierra y obtener mejores beneficios desde el punto de vista financiero (Vilca y González, 2011). Un tercer grupo de posibles inversionistas son aquellos que viven en el medio rural, poseen recursos financieros para invertir en agricultura tecnificada y de alto rendimiento pero que no poseen tierras ni maquinaria. En esta última situación también permanecen en el medio urbano personas con capacidad financiera e interés de invertir en el campo.

Es totalmente cierta la afirmación de que existen productores que tienen tierra, pero no tienen maquinaria ni recursos financieros para tomarla en renta, existen otros que tienen maquinaria, pero no tienen suficiente tierra ni capital y un tercer caso de poseedores de recursos financieros pero que tienen poca o nada de tierra y tampoco cuentan con maquinaria. Que no cuentan ni con tierra ni con maquinaria.

Se puede afirmar con muy buen sustento, que la integración de los tres recursos productivos llevaría a una explotación agrícola de mejores resultados para cada uno de los participantes porque se trataría de una actividad agrícola con uso de mayores y mejores insumos productivos sobre el recurso tierra (Portillo et al., 2020).

Cada uno de los tres sectores pueden ser a la vez un grupo de participantes como sería el caso de los poseedores de tierra, dado que la de uno sólo sería muy pequeña, a la vez puede dividirse la sección capital en capital y mano de obra si esta se encuentra, como se da frecuentemente, abundante entre los poseedores de tierra (González et al., 2014).

Ante esta situación, se propone formar asociaciones temporales de participantes inversionistas de sus recursos en una misma explotación agrícola que beneficiaría a los poseedores de estos recursos. Esta es una alternativa a la de formar cooperativas agrícolas de productores en las que las experiencias en México son de nada buenos resultados entre otros una actual resistencia de los productores para participar en cooperativas.

Investigaciones como de Guerrero $(1989,1998)$ refiere a que a propuesta de formar asociaciones de inversionistas conlleva beneficios adicionales como el de mejorar las condiciones de venta de los productos si en esta parte del negocio participan directamente los socios de capital quienes 
seguramente poseen mejores condiciones para negociar precio de venta, de acarreo del producto hacia los lugares más cercanos al mercado de consumidores e incluso incorporar de forma adicional y como parte del negocio, la agregación de seguro agrícola o valor agregado a la mercancía procesando el producto en instalaciones agroindustriales en la periferia de los terrenos productivos.

De esta forma se evitaría el gran margen de precios que actualmente existe entre precio pagado al productor y precio pagado al consumidor final, precio del cual sólo una pequeña proporción llega al productor y el resto se queda en manos de los que ejecutan compra en campo, almacenamiento, transporte e industrialización del producto y en mucho menos tiempo que lo que le lleva al productor desde que siembra hasta que cosecha (Guerrero, 1993a). La participación de socios de capital seguramente proporciona mejores condiciones negociadoras para evitar los abusos históricos que se realizan en contra de los pequeños productores.

Estos y otras ventajas más estarían mejorando la situación de todos los participantes en la asociación de pool agrícola o como se le quiera llamar a este tipo de asociación, así lo refiere Beneke (1991). Experiencias en otros países como Argentina donde estos grupos eventuales y de tiempos de duración de un solo ciclo agrícola y con repeticiones con cambios en las siguientes temporadas de siembra. En este país se conoce como pool agrícola (Súper Campo, 2012).

\section{Problema para resolver}

El propósito principal del presente escrito es el de aportar un procedimiento aceptable de cómo repartir el beneficio de producto que se genera en una explotación agrícola en la que participan como socios más de una fuente de inversión en el proceso, pero es un tema íntimamente ligado a la salida del problema de producción de baja rentabilidad de productores agrícolas pequeños produciendo con sus escasos recursos. El objetivo central es como resolver el problema de reparto de utilidades de forma satisfactoria para resolver los siguientes problemas.

En una producción en sociedad lo que ya representa un avance y mejora significativa para producción en el campo, se puede presentar una situación en la que cada participante argumente que el recurso que él aporta es el más importante y por lo tanto debería recibir la mejor retribución, los dueños del recurso tierra pueden afirmar que sin tierra no hay negocio, por su parte el dueño de maquinaria afirmará que sin el uso de maquinaria y con sólo insumos tradicionales no se obtiene cosecha con valor suficiente para pagar los costos y proporcionar una ganancia, mientras que el dueño del capital dirá que sin capital para invertir en insumos mejorados, la producción no puede reportar ganancias necesarias para justificar la inversión, así lo observa Cañas (1991).

El presente documento presenta una forma de repartir el valor de la producción entre los diferentes participantes de forma justa y basada en una solución obtenida por procedimientos de administración técnica, se calcula qué cantidad o parte del valor de la producción corresponde a cada uno de los participantes (Romero, 1998). El procedimiento consiste en identificar que aportación hace cada uno de los recursos empleados a la producción física y monetaria de las cosechas. Para lo cual se utiliza concretamente, la programación lineal.

En una misma superficie sembrada con participación de socios poseedores de los recursos para producir con mejor eficiencia de la inversión se tienen mejores resultados que trabajando como se viene haciendo actualmente. Esta forma de practicar la agricultura favorece no sólo con mayores 
cantidades de beneficios para los productores sino también mayores cantidades de productos alimenticios que estarán disponibles en el mercado. Con metodología de programación lineal es posible identificar con precisión qué proporción de la producción y de su valor comercial le corresponde a cada uno de los participantes en la producción de un pool agrícola (Buongiernos y Gilless, 2003).

Los objetivos fueron elaborar un procedimiento de reparto de utilidades en cuyo procesamiento de información y datos, para evitar prejuicios e influencia personal de los participantes; a través, de intervenciones de discusión para repartir el producto que se genere en la asociación.

Explicar en forma fácil y operativa, la forma de obtener los datos de la cantidad de producto que corresponde a cada uno de los grupos o personas participantes mediante un procedimiento de programación lineal aplicada. Procedimiento de esta disciplina a la que se le ha agregado un ajuste que significa aportación al manejo de la programación lineal, para lograr el propósito mencionado. Combinar procedimientos de selección de cultivos y sus respectivas áreas a sembrar para obtener los máximos beneficios posibles, empleando las cantidades de recursos aportadas por los grupos de inversionistas participantes y la identificación de cantidades de producto o valor de la producción que le corresponde a cada uno de estos socios (Bishop y Toussaint, 1996).

\section{Antecedentes}

Señalando otras formas que actualmente se utilizan y comparando procedimientos. A falta de conocimiento de procedimientos respaldados con teoría y procedimientos econométricos aplicables al problema de distribución de ingresos en una empresa colectiva, con frecuencia, los ingresos de cada uno de los participantes, se decide por imposición de cada uno de ellos de cuanto quiere obtener como retorno de su inversión.

De esta forma, por ejemplo, los dueños de la tierra pueden reclamar una renta fija de este recurso, a pagar por adelantado y no le interesa lo que se obtenga de ingresos. En estos casos eliminan riesgos, pero renuncian a beneficios adicionales de ganancia en el reparto de utilidades y al resto de participantes les cancela la seguridad de seguir contando con la tierra a un costo razonable en años futuros (Clop y Juárez, 2003).

Lo mismo puede suceder con los poseedores de recursos monetarios sin tener tierra suficiente para invertir su potencial financiero que sólo esté dispuesto a invertir en calidad de préstamo con intereses y asegurar su monto de ganancia esperada en el nivel que deciden a través de la tasa de interés. Los poseedores de maquinaria con capacidad sobrada para explotar su propio terreno, puede tener la posición de que presta sus servicios de mecanización y que le estén retribuyendo un pago en cuanto se realice cada una de las actividades agrícolas en las que se le requiere (Romero, 1989; 1998).

Para los tres casos mencionados puede ser atractivo que trabajando en sociedad los tres recursos productivos aumentan el potencial de producción que significa el convertir, por este medio, hacia una producción tecnificada de mayores resultados de producto a repartir, los poseedores de maquinaria tendrán la posibilidad efectiva de recuperar en menor tiempo y con mejor retribución el costo de su maquinaria y adicionalmente la ventaja que tendrían al enfrentar las condiciones de precio de venta del producto que no tienen por si solos los productores poseedores de la tierra. Este 
modelo presentado con únicamente tres sectores de participantes puede modificarse de tal forma que se considere por separado un factor mano de obra si así conviniera y se contara por separado la mano de obra proporcionada por los dueños de la tierra (Romero, 1993a).

También es posible qué en una unidad de riego, el agente que controla el uso del agua puede participar como un integrante de la sociedad y estar de acuerdo en que su retribución se le entregue al término de las actividades cuando se venda el producto. También es posible qué en uno de los integrantes mencionados como los poseedores de maquinaria, no estén de acuerdo en esperar al final del ciclo para obtener su ingreso, en este caso este servicio pasaría a formar parte del capital y sería el factor capital quien aporte estos pagos aumentando así su participación y sus ingresos al final del proceso de producción (Cartier y Cartier, 2003).

Todas estas variantes y otras más pueden darse y es importante mencionarlas porque representa una flexibilidad del modelo. En otra forma de mencionarlo, este modelo presentado de forma simplificada para su mejor comprensión puede modificarse en su número de grupos participantes como inversionistas lo que le da mayores posibilidades de ser aplicado manteniendo como constante que el reparto de utilidades se dará de acuerdo con la aportación que hagan, a la generación de producto, cada uno de los recursos empleados.

\section{Materiales y métodos}

\section{El procedimiento para emplear}

Un ejemplo simplificado elaborado con fines de fácil entendimiento del procedimiento a seguir es el modelo con las siguientes características: empleando valores hipotéticos, pequeños y en números redondos para seguir fácilmente la exposición y no perderse en operaciones complejas que afecten la comprensión.

Para mantener la característica de procedimiento simple para su fácil comprensión, se desarrolla uno donde sólo aparecen tres factores productivos los cuales pueden, en un caso real, dividirse en más factores; por ejemplo: si los dueños de la tierra mencionan que ellos aportarían la mano de obra, esta se trataría como otro factor a retribuir, de lo contrario si se acuerda que a los que aporten mano de obra se les paga directamente con el salario vigente en el lugar, el rubro pasará a ser parte del capital invertido y su retribución entre los participantes será para el que aporte el capital. Puede ser que el pool se realice en un área de dominio de un sistema de riego y el que posee los derechos del agua los aporte para el pool y reclame su retribución que le corresponda. Una opción, sería que el que proporciona el capital se encargue de pagar este servicio y pasa a ser parte de la inversión de capital y por tanto se hace acreedor a lo que resulte en el reparto del valor de la producción.

Modelo explicativo con tres actividades agrícolas, tres recursos o insumos a emplear y sus cantidades disponibles. Se dispone de 12 ha de tierra cultivable; $48 \mathrm{~h}$ máquina; 24 unidades monetarias de capital. Los cultivos posibles son maíz, trigo y sorgo que generan 400, 300 y 200 unidades monetarias por hectárea, respectivamente. Si se planea cuantas hectáreas de cada cultivo se deben sembrar conociendo los ingresos que genera cada hectárea de cada cultivo -precios netosempleando los recursos disponibles y conociendo que cantidad de cada uno de los recursos se requiere para cada una de las hectáreas a cultivar -coeficientes técnicos- y persiguiendo que el valor de las cosechas sea el máximo posible, se realiza el procedimiento como lo refiere el Cuadro 1. 
Cuadro 1. Precios netos, coeficientes técnicos y recursos disiblespon.

\begin{tabular}{ccccc}
\hline \multirow{2}{*}{ Cultivo (ha) } & \multirow{2}{*}{$\begin{array}{c}\text { Precios netos } \\
\left(\$ \text { ha }^{-1}\right)\end{array}$} & Tierra $($ ha $)$ & Maquinaria $(\mathrm{h})$ & ${\text { Capital }\left(\$ \text { ha }^{-1}\right)}^{n}$ \\
\cline { 3 - 5 } & 400.00 & 1 & 6 & 36.00 \\
Maíz (X1) & 300.00 & 1 & 6 & 24.00 \\
Trigo (X2) & 200.00 & 1 & 2 & 18.00 \\
Sorgo (X3) & & 12 & 48 & 324.00 \\
Recursos disponibles & & &
\end{tabular}

Beneke (1991) con datos modificados. Donde: X1, X2 y X3 son hectáreas para sembrar de cada cultivo 1, 2 y 3 respectivamente.

Para su procesamiento, estos datos se ingresan a un programa informático de programación lineal. Solicitando la solución de cuantas hectáreas deben sembrarse de cada uno de los cultivos factibles para obtener el máximo beneficio. En el programa LINDO (1996) se arreglan y se incorporan a la página que aparece al abrir este paquete computacional en esta forma.

MAX 400X1+300X2+200X3

SUBJEC TO

$\mathrm{X} 1+\mathrm{X} 2+\mathrm{X} 3<=12$

$6 \mathrm{X} 1+6 \mathrm{X} 2+2 \mathrm{X} 3<=48$

$36 \times 1+24 \times 2+18 \times 3<=324$

Donde se solicita maximizar el valor de la producción sabiendo que cada hectárea de X1, X2 y X3 generan 400, 300 y 200 unidades de dinero por cada hectárea sembrada respectivamente. Sujeto a las siguientes restricciones.

Que la superficie programada sea igual o menor que la cantidad disponible de 12 ha. Que las horas máquina empleadas sean iguales o menores que las 48 horas disponibles. Que el capital total a aplicar sea menor o igual a la cantidad disponible 324.

Los coeficientes que aparecen en esta desigualdad son la cantidad de recurso que utiliza cada hectárea de cada uno de los cultivos a sembrar. Los resultados son obtenidos en una tabla de información de la siguiente forma: donde los resultados generados más importantes para el propósito perseguido en este caso son: el dato que aparece como OBJECTIVE FUNCTION VALUE 3600 corresponde al valor total de la producción que se genera.

En seguida aparecen tres columnas de las cuales las dos primeras con nombre de VARIABLE y VALUE señalan que se deben cultivar 6 ha de maíz, cero de trigo y 6 ha de sorgo para obtener el mayor valor de la producción. En el siguiente bloque de datos, la columna de precios sombra que aparece como DUAL PRICES. Estos últimos datos señalan el valor que genera cada unidad de recurso empleado para obtener el valor total de la producción. Los precios sombra son: 25, 12.5 y 8.333333 para tierra, maquinaria y capital respectivamente.

Si se multiplica cada uno de estos precios sombra por la cantidad de unidades de su respectivo recurso que entró en el proceso de producción, se obtiene el valor que genera cada recurso. recurso tierra $=25 \mathrm{X} 12$, maquinaria $=12.5 \mathrm{X} 48$ y capital $=8.333333 \mathrm{X} 324$. El resultado es la parte del valor 
total de la producción que le corresponde a cada participante por concepto de uso de su recurso aportado. Sumando las aportaciones de cada recurso, puede comprobarse que se obtiene el valor total de la producción a repartir $=3600$. Los resultados se presentan en el Cuadro 2.

Cuadro 2. Contribución al valor de la producción por parte de cada recurso empleado.

\begin{tabular}{cccc}
\hline Recurso & Cantidad empleada (a) & Precio sombra (b) & $\begin{array}{c}\text { Contribución al valor de } \\
\text { la producción (axb) }\end{array}$ \\
\hline Tierra & $12 \mathrm{ha}$ & 25 & 300 \\
Maquinaria & $48 \mathrm{~h}$ & 12.5 & 600 \\
Capital & 324 unidades de $\$$ & 8.333333 & 2700 \\
Valor total & & & 3600 \\
\hline
\end{tabular}

\title{
Hoja de salida del proceso de cómputo
}

LP optimum found at step 3

Objective function value

1) 3600.000

\author{
VARIABLE, VALUE, REDUCED COST \\ X1 6.00 .0 \\ $\mathrm{X} 20.00 .0$ \\ X3 6.00 .0
}

ROW SLACK OR SURPLUS DUAL PRICES

2) 0.025 .00

3) 0.012 .50

4) 0.08 .33

No. Iterations $=3$

Ranges in which the basis is unchanged

Rangos en los cuales la solución básica no cambia

Coeficientes de la función Objetivo

Variable, Current, Allowable, Allowable

Coef, Increase, Decrease

X1 40020.0099 .99

X2 30099.933 .33

X3 20050.0016 .66

Righthand Side Ranges

Row current allowable allowable

Rhs, Increase, Decrease

212.0000004 .8000000 .000000

348.00000024 .0000000 .000000

4324.0000000 .00000072 .000000 


\section{Resultados y discusión}

\section{Advertencia de primera importancia}

Los resultados anteriores se obtienen en dos ejecuciones del programa informático, el primer modelo que se ensaya es utilizando los datos de cantidades totales de recurso que cada participante está dispuesto a aportar.

Obteniendo los resultados del programa es altamente probable que la combinación de cantidades de recursos disponibles para emplear y obtener el máximo ingreso, no se consuman en su totalidad algunos de estos recursos por falta de otros recursos que los complementen (Beneke, 1992). En estos casos, en los precios sobra de los recursos que no entran en su totalidad a la solución, el programa les asigna un precio sobra de cero.

El principio teórico que se manifiesta es que un recurso que sobra no es escaso y por lo tanto el programa le asigna valor de cero a precio sombra. El proceso para arreglar este detalle es volver a correr el programa reduciendo la cantidad sobrante de cada uno de los recursos que tienen este sobrante y que aparecen en la tabla de resultados. De tal forma, que se corre el programa nuevamente con las cantidades que entraron a la solución como si fueran la totalidad de recurso con que se cuenta y de esta manera se convierten todos los recursos en recursos escasos y el programa les asigna el valor correspondiente de precio sombra para emplearlo en la distribución del valor de la producción que se genera (Arzeno y Ponce, 2014; Romero, 1993a).

La explicación de este detalle consiste en que el precio sombra se emplea también para otros fines entre los cuales el precio sombra se identifica como el valor que se estaría dispuesto para adquirir y agregar una unidad más de recurso para ser incorporado a la producción. Si el precio sombra es de valor cero es porque ese recurso no es limitativo en el programa, por lo tanto, si es una sola empresa la que invierte todos los recursos, no estaría dispuesto a pagar por agregar una unidad más de recurso para incorporarlo a su producción si ese recurso resulta ser abundante con respecto al resto de factores de la producción incluidas en el modelo (Maiztegui, 2009).

Si uno de sus recursos ingresa en su totalidad en el programa de cultivo y por lo tanto aparece un precio sombra de valor mayor al que pagaría por incorporar una unidad más de ese recurso que puede adquirir, puede optar por tomar esa unidad de recursos para incorporarlos a la explotación con la seguridad de que el valor de la producción aumentaría en la magnitud señalada por el precio sombra.

El precio sombra en el modelo básico que posee el programa informático LINDO (1996), señala en cuanto aumentaría el valor de la producción si se incorpora una unidad más del recurso en cuestión. Es obvio que si el precio sombra es cero es porque le sobra ese recurso en el monto disponible y no estaría dispuesto a adquirirlo fuera de la empresa en unidades adicionales para incorporarlo al plan de producción (Manzanal, 2014).

Puede verificarse lo mencionado en el párrafo anterior, corriendo el modelo diseñado sustituyendo el valor del capital disponible de 234 por el de 360, así lo demuestra (Tourer, 1986; Romero, 1993b). La solución de cuantas hectáreas de cada cultivo debe sembrarse, será la misma que en el 
actual modelo ensayado, pero del capital de 360 la tabla de resultados señalará que este recurso tiene un precio sombra de cero que indica que sobra una cierta cantidad de capital y no sería conveniente pagar por obtener una unidad de recurso adicional.

Es importante mencionar, qué tratándose de diferentes dueños de los recursos participativos, qué si en la primera corrida del programa algunos recursos 'salen sobrando' y por tanto sus precios sombra son de cero, quien los invierte no estaría de acuerdo en invertirlos si no le corresponde ningún valor en el reparto de utilidades. De ahí la importancia de correr el modelo por una segunda ves programando con cantidades de recursos disponibles en las cantidades que entran en la primera solución y no en las cantidades disponibles (Davis y Johnson, 1987). Dicho de otra forma, se corre el programa por primera vez con todas las cantidades de cada recurso puestas a disposición del negocio y en una segunda corrida se aplican como cantidades de recursos máximos, en la cantidad en que entraron al proceso de optimización para generar el máximo valor de la producción.

El segundo programa informático se ejecuta reduciendo las cantidades disponibles de los recursos restando el sobrante que se obtiene en la primera solución. La salida de resultados del primer ensayo también contiene datos de cuánto sobra de cada uno de los recursos disponibles para cada uno de los recursos. En la columna de SLACK OR SURPLUS.

Este mecanismo es la aportación por parte del presente documento, que hasta ahora no se encuentra en ningún documento de programación lineal, sin desconocer qué el paquete proporciona muchos más indicadores que permiten tomar decisiones sobre modificaciones en el plan de inversión como puede apreciarse en la hoja de salida de la computadora presentado páginas atrás (Manzanal, 2014; Ballesteros, 1992). El tratamiento del programa es por lo tanto exclusivo para un problema de administración en el que participan varios inversionistas y que se requiere saber cuánto le corresponde del ingreso generado sin recurrir a discusiones donde cada inversionista considera que su recurso aportado es el de mayor importancia y que por lo tanto le corresponde una mayor cantidad de recurso (Castillo, 2013).

Véase la siguiente hoja de salida de la computadora los resultados de 'precios sombra' (DUAL PRICES) y cantidades de superficie a sembrar del modelo en las columnas VARIABLE y VALUE que señalan $\mathrm{X} 1=6$ hectáreas del primer cultivo, cero del segundo $(\mathrm{X} 2=0)$ y del tercero $(\mathrm{X} 3=6)$ los mismos resultados y para el precio sombra del recurso capital considerando disponibles 360 unidades, su precio sombra es cero.

Lp optimum found at step 2

Objective function value

Valor de la producción a generar

1) 3600.000

Variable, Value, Reduced cost

$\mathrm{X} 16.0000000 .000000$

X2 0.000000100 .000000

X3 6.0000000 .000000

Recursos sobrantes Precios sombra

Row slack or surplus dual prices 
2) 0.000000100 .000000

3) 0.00000050 .000000

4) 36.0000000 .000000

Ranges in which the basis is unchanged:

Rangos en los precios netos (ingresos por hectárea) donde la solución básica no cambia.

OBJ Coefficient ranges

Variab current, Allowable, Allowable

Coef, Increase, Decrease

X1 400200100

X2 300100 Infinity

X3 20020066.6

Righthand side ranges

ROW Current Allowable Allowable

RHS, Increase, Decrease

21244

348824

4360 INFINITY 36

\section{Conclusiones}

La programación lineal aplicada a grupos de inversionistas en la producción agrícola, aplicando el presente procedimiento, evitan discusiones en la toma de acuerdos de cómo repartir el valor de la producción que se genera en la inversión que se realiza. El reparto de utilidades siguiendo el presente procedimiento se define por la aportación que efectúa cada recurso en la generación del valor del producto. Este modelo constituye una buena salida para activar en las mejores condiciones a los programas del Gobierno Federal de mecanización del campo rural que no han dado los resultados esperados porque la maquinaria pertenece a un pequeño grupo de productores y éstos tienen serias dificultades para emplear toda su capacidad porque tienen poca tierra y el resto de los productores no tienen recursos para tomarlos en renta.

El procedimiento aplicado en la práctica es adecuado para evitar la violación de las leyes agrarias como el de la renta de la tierra ejidal por largo tiempo, la cual puede permanecer en manos de sus poseedores y explotarla en mejores condiciones que en su forma habitual. El mecanismo de producción en grupos de inversionistas en acciones acordadas para una sola cosecha por separado da oportunidad a los dueños de maquinaria el recuperar la amortización de su inversión en este rubro lo que no puede obtener sembrando la reducida superficie de su propiedad ni por renta de la maquinaria ante tenedores de tierra que no disponen de recursos para tomarla en renta. Este tipo de organización para la producción reduce considerablemente el temor y desconfianza que tienen los pequeños productores emanadas de otro tipo de organizaciones como las de cooperativas agrícolas.

La presente sugerencia de formación de asociaciones para la producción es aplicable a una gran cantidad de áreas geográficas del país donde existen las situaciones descritas entre productores y tenedores de otros recursos productivos para la agricultura. La intervención del Gobierno Federal o Estatal no es reclamada dado que normalmente en cada situación de agricultura tradicional o de 
tecnología muy limitada existen en esa misma área o en lugares cercanos, recursos tecnológicos, capital y capacidad de administración que sólo tienen que integrarse para generar con mejor tecnología mayores y mejores cantidades de productos del campo. Por lo tanto, la participación gubernamental sería marginal. En la exposición de la presente propuesta no se menciona la participación del gobierno, al menos no de participación directa, sino sólo de promotor y difusor a través de sus instancias representativas en las áreas productoras agrícolas.

\section{Recomendaciones}

Difundir este procedimiento en el sector rural para adoptar el procedimiento de distribución del producto que se genere a través de asociaciones de inversionistas en el sector rural del país.

Promover estas asociaciones como una medida para complementar los programas de mecanización de la agricultura que no han tenido efecto muy positivo hasta ahora, pero pueden reactivarse con mejores perspectivas de éxito si se considera la participación de poseedores de maquinaria en estos grupos de inversionistas. Asignar el nombre de pool de siembra, para que los interesados en revisar antecedentes encuentren referencias bibliográficas sobre estas agrupaciones de socios. De los cuales pueden aprovechar experiencias y detalles que puedan ser adaptados y agregados a los grupos propuestos en el presente escrito. Cada pool agrícola que se forme tendrá seguramente interesados en conocer los mecanismos de funcionamiento de los grupos que ya actúan en otras áreas de la producción y en otros países.

Se sugiere promover por parte de las instancias gubernamentales este tipo de asociaciones en el campo, principalmente en las áreas de grandes números de productores cada uno de ellos con pequeñas superficies de tierra. Como elemento de convencimiento, afirmar y asegurar que el reparto de utilidades entre participantes es lo más justo y recomendable para resolver el problema de distribución de ingresos generados en la producción. Promocionar estas asociaciones haciendo eco de que difieren de forma significativa de las que antecedieron como las cooperativas agrícolas que se recuerdan con no gratos resultados. Que sean los participantes inversionistas quienes decidan las actividades de administración y de preferencia integrantes inversionistas del grupo.

\section{Literatura citada}

Arzeno, M. y Ponce, M. 2014. Las contradicciones de las políticas públicas: desarrollo territorial rural en la provincia de Misiones, Argentina. Cuadernos del Cendes. 31(85):69-93.

Ballestero, E. 1992. Principios de economía de la empresa. Alianza Universidad Textos, Madrid, España. 644-651 pp.

Beneke, R. R. 1991. Programación lineal aplicada a la agricultura. Iowa State University. ISBN: 9788470032646. (Ed.) Aedo. USA. 222 p.

Bishop, C. y Toussaint, W. 1966. Introducción al análisis de economía agraria. Limusawiley. (Ed.). México, DF. 262 p.

Buongiorno, J. and Gilless, J. K. 2003. Decision methods for forest resource management. Academic Press, San Diego. 439 p.

Cañas, J. A. 1991. Programación líneal: determinación de coeficientes de la submatriz de sucesión, cuando intervienen cultivos plurianuales. Investigación Agraria. 6(1):5-17. 
Cartier, E y Cartier, J. 2003. Tambos-análisis de sus procesos de producción con fines de costeo. In: XXVII Congreso Argentino de Profesores Universitarios de Costos. Tandil, Argentina. $27 \mathrm{p}$.

Castillo, Y. 2013. Adaptación de un modelo para caracterizar los procesos de gestión de la innovación en las empresas del sector de las TIC de la ciudad de Popayán. Rev. Electrónica Gestión de las Personas y Tecnología. Universidad de Santiago de Chile. Santiago de Chile.

Clop, M. M. and Juárez, F. 2003. Programació lineal per a l'enginyeria agrària. Casos pràctics. Edicions de la Universitat de Lleida. 220 p.

Davies, L. and Johnson, K. N. 1987. Forest management. (Ed.). McGraw Hill, New York. 790 p.

González, C. J.; Coliñanco, G. L.; Caro, C. L. y Romero, H. N. 2014. Estrategia y cultura de innovación, gestión de los recursos y generación de ideas: prácticas para gestionar la innovación en empresas. Universidad del Norte. Rev. Pensamiento y Gestión. 36:109-135.

Guerrero, A. 2011. Innovación: clave para el éxito de la gestión empresarial, en la micro, pequeña y mediana empresa. Rev. Nacional de Administración. 74 edición julio-diciembre. Costa Rica. Universidad Estatal a Distancia. 2(2):61-80.

Portillo, V. M.; Sangerman-Jarquín, D. Ma.; Ayvar, V. Ma. del R. y Ramírez, J. R. 2020. Modelo de aseguramiento agrícola privado para pequeños productores agrícolas en México. Rev. Mex. Cienc. Agric. 11(6):13889-1402.

LINDO. 1996. Paquete computacional Linear, Integer, Nolineasr and O. USA.

Maiztegui, M. H. 2009. Una nueva modalidad asociativa en Argentina: el pool de siembra, Argentina. Revista Estudios Agrarios. 15(41):149-172.

Manzanal, M. 2014. Crisis, especulacion y desigualdad en América Latina. Las nuevas formas de valoración del capital y de producción del territorio frente a la problemática del hambre y la desnutrición. Revista de Ciencias Sociales. Segunda época. Primer semestre núm. 25.

Romero, C. 1982. Modelos económicos en la empresa. (Ed.). Mundi-Prensa. 25(2):157-315.

Romero, C. 1989. Modelos de planificación forestal: una aproximación al análisis multicriterio. Revista de Estudios Agrosociales. 147:71-92.

Romero, C. 1993a. Técnicas de gestión de empresas. Ed. Mundi Prensa, Madrid. España. 306 p.

Romero, C. 1993b. Teoría de la decisión multicriterio: conceptos, técnicas y aplicaciones. Ed. Alianza Universidad Textos, Madrid. 341 p.

Romero. C. 1998. Evaluación financiera de inversiones agrarias. Ediciones MundiPrensa. 78 p.

Súper Campo. 2012. Cómo elegir un fideicomiso agropecuario. Debido a la rentabilidad del negocio agropecuario ha crecido la oferta de pools de siembra. Economía y Mercados. 2 p.

Tourer, L. 1986. Risk preferences of dairy farmers. North Central. J. Agric. Econ. 8(1):7-15.

Vilca, G. y González, J. 2011. Centro de política y gestión de la innovación y el emprendimiento tecnológico, un espacio de desarrollo transversal en la Facultad Tecnológica de la USACH. Rev. Electrónica Gestión de las Personas y Tecnología, 12. Universidad de Santiago de Chile. 